Physics

Physics Research Publications

Purdue University

Year 2005

\title{
Resonant electronic raman scattering in a Van Vleck II-VI diluted magnetic semiconductor: Cd1-xFexTe
}
S. Tsoi
I. Miotkowski
S. Rodriguez
A. K. Ramdas
H. Alawadhi
T. M. Pekarek

This paper is posted at Purdue e-Pubs.

http://docs.lib.purdue.edu/physics_articles/243 


\title{
Resonant electronic Raman scattering in a Van Vleck II-VI diluted magnetic semiconductor: $\mathrm{Cd}_{1-x} \mathrm{Fe}_{x} \mathrm{Te}$
}

\author{
S. Tsoi, I. Miotkowski, S. Rodriguez, and A. K. Ramdas \\ Department of Physics, Purdue University, West Lafayette, Indiana 47907, USA \\ H. Alawadhi \\ Department of Basic Sciences, University of Sharjah, United Arab Emirates \\ T. M. Pekarek \\ Department of Chemistry and Physics, University of North Florida, Jacksonville, Florida 32224, USA
}

(Received 13 May 2005; published 28 October 2005)

\begin{abstract}
The Van Vleck paramagnetism of $\mathrm{Cd}_{1-x} \mathrm{Fe}_{x} \mathrm{Te}$, a diluted magnetic semiconductor, is explored with electronic Raman spectroscopy of an internal transition of $\mathrm{Fe}^{2+}$, on the one hand, and the spin-flip Raman scattering (SFRS) from donor-bound electrons, on the other. Zeeman splitting of the Raman transition from the nonmagnetic ground state to the first excited state displays patterns consistent with energy levels responsible for the Van Vleck paramagnetism. SFRS, in turn, delineates characteristic features of the Van Vleck magnetization, as expected from $s-d$ exchange interaction. The combination of SFRS and magnetization measurements yielded the $s-d$ exchange constant in $\mathrm{Cd}_{1-x} \mathrm{Fe}_{x} \mathrm{Te}, \alpha N_{0}=244 \pm 10 \mathrm{meV}$.
\end{abstract}

DOI: 10.1103/PhysRevB.72.155207

PACS number(s): 78.30.Fs, 78.20.Ls, 71.70.Gm, 71.70.Ej

\section{INTRODUCTION}

The remarkable co-existence of semiconducting and magnetic properties of the tetrahedrally coordinated II-VI diluted magnetic semiconductors (DMSs) - the $\mathrm{II}_{1-x} M_{x} \mathrm{VI}$ ternary alloys- $(M \equiv 3 d$ transition-metal ion, i.e., $3 d$ TMI) has been extensively documented in the context of their striking magnetic and magneto-optic phenomena. ${ }^{1}$ The fabrication of $\mathrm{In}_{1-x} \mathrm{Mn}_{x}$ As epilayers ${ }^{2}$ by molecular-beam epitaxy extended the field to III-Vs and has triggered the current intense interest in spintronic materials. ${ }^{3}$

The remarkably large excitonic Zeeman effect and the associated Faraday and Voigt effects ${ }^{4}$ and the spin-flip Raman shifts of donor-bound electrons displayed by DMSs result from the spin-spin $s p-d$ exchange interaction of the $3 d$ electrons of the TMIs with the $s$ electrons of the conduction band and the $p$ electrons of the valence band. Crystal-field effect, spin-orbit coupling, and static or dynamic Jahn-Teller effects are significant microscopic mechanisms which determine the thermal average of the magnetic moment of the specific $3 d$ TMI in the presence of an external magnetic field. ${ }^{5,6}$ The variety of magnetic behavior as one proceeds from $\mathrm{Sc}^{2+}$ to $\mathrm{Ni}^{2+}$ has been theoretically analyzed in terms of the above mechanisms. For example, $\mathrm{Mn}^{2+}$, with its negligible crystalfield splitting in the DMSs, has, to a good approximation, an "atomiclike" ${ }^{6} S_{5 / 2}$ ground state with an effective spin of $(5 / 2) \hbar$; hence the corresponding magnetization displays a $B_{5 / 2}$ Brillouin function behavior. In contrast, $\mathrm{Co}^{2+}$ in $\mathrm{Cd}_{1-x} \mathrm{Co}_{x} \mathrm{Te}$ possesses a ground state with an effective spin of $3 / 2$, resulting from crystal-field and spin-orbit effects, which leads to a $B_{3 / 2}$ magnetization behavior. We recently showed ${ }^{7}$ that vanadium enters $\mathrm{CdTe}$ as $\mathrm{V}^{2+}$, also exhibiting a $B_{3 / 2}$ behavior. The case of $\mathrm{Fe}^{2+}$ is more complex. The ${ }^{5} D(L=2, S=2)$ ground state of the $\mathrm{Fe}^{2+}$ free ion is split by $\mathcal{H}_{C F}$, the crystal field of $T_{d}$ symmetry, into a ${ }^{5} \Gamma_{3}$ orbital doublet and a higher ${ }^{5} \Gamma_{5}$ orbital triplet (Fig. 1), separated by $\Delta$. Spin-orbit interaction $\mathcal{H}_{S O}=\lambda \mathbf{L} \cdot \mathbf{S}$, taken in the first and the second order, yields the level structure ${ }^{6}$ with a nonmagnetic $\Gamma_{1}$ singlet ground state and several closely lying magnetic excited states. Inclusion of the dynamic Jahn-Teller effect, known to occur for $\mathrm{Fe}^{2+}$, results in nonmagnetic, ground vibronic levels. ${ }^{8,9}$ At the lowest temperatures, only the nondegenerate electronic ground state is occupied, so that $\mathrm{Fe}^{2+}$ has no permanent magnetic moment in the crystal. Under application of an external magnetic field $\mathbf{H}$, however, the Zeeman interaction $\mathcal{H}_{Z}=\mu_{B} \mathbf{B} \cdot(\mathbf{L}+2 \mathbf{S})$ mixes $\Gamma_{1}$ with the higher-lying levels, inducing a magnetic moment along $\mathbf{H}$. This behavior has been referred to as Van Vleck paramagnetism.

The energy levels of $\mathrm{Fe}^{2+}$ in different II-VI's have been studied with optical absorption, ${ }^{10-12}$ low-field magnetic susceptibility, ${ }^{13}$ and magnetization. ${ }^{14}$ Raman spectroscopy has played an important role in the detailed characterization of the internal transitions of $\mathrm{Fe}^{2+}$ from its $\Gamma_{1}$ ground level to lowest excited levels in $\mathrm{Cd}_{1-x} \mathrm{Fe}_{x} \mathrm{~S},{ }^{15} \mathrm{Cd}_{1-x} \mathrm{Fe}_{x} \mathrm{Se},{ }^{16}$ and $\mathrm{Cd}_{1-x} \mathrm{Fe}_{x} \mathrm{Te},{ }^{17}$ including splittings in an external magnetic field.

The exchange interactions between $\mathrm{Fe}^{2+}$ and band carriers in various DMSs have been determined with magneto-optic techniques. Excitonic Zeeman effect observed in reflectivity together with magnetization measurements yielded the $s-d$ exchange constant $\left(\alpha N_{0}\right)$ and the $p$ - $d$ exchange constant $\left(\beta N_{0}\right)$ in $\mathrm{Zn}_{1-x} \mathrm{Fe}_{x} \mathrm{Se},{ }^{18} \mathrm{Cd}_{1-x} \mathrm{Fe}_{x} \mathrm{Se},{ }^{19} \mathrm{Cd}_{1-x} \mathrm{Fe}_{x} \mathrm{Te},{ }^{20}$ and $\mathrm{Zn}_{1-x} \mathrm{Fe}_{x} \mathrm{Te}^{21}$ Alawadhi et al. ${ }^{22}$ obtained these exchange constants for $\mathrm{Cd}_{1-x} \mathrm{Fe}_{x} \mathrm{Te}$ from the excitonic Zeeman effect experiment carried out with wavelength-modulated reflectivity with a precision better than that in Ref. 20. Faraday effect has yielded the difference $(\alpha-\beta) N_{0}$ in $\mathrm{Cd}_{1-x} \mathrm{Fe}_{x} \mathrm{Te} .^{23,24}$

Unlike excitonic Zeeman effect and Faraday rotation, which reflect the combined Zeeman splitting of the conduc- 


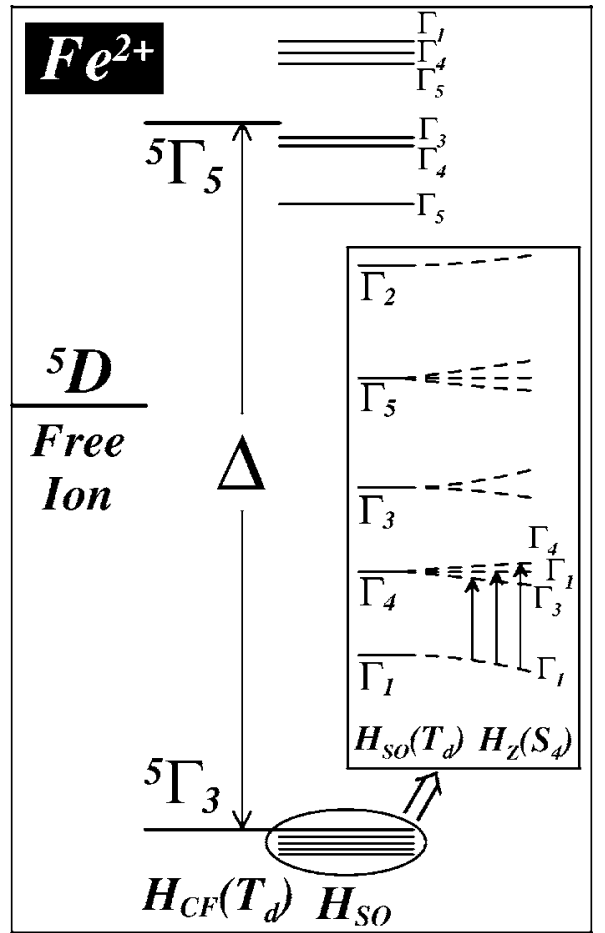

FIG. 1. Energy levels of $\mathrm{Fe}^{2+}$ in $T_{d}$ crystal field $\left(H_{C F}\right)$ with the spin-orbit $\left(H_{S O}\right)$ and Zeeman $\left(H_{Z}\right)$ interactions taken into account. Inset: Magnetic field along [001] reduces the $T_{d}$ site symmetry of $\mathrm{Fe}^{2+}$ to $S_{4}$. Vertical arrows show the internal transitions studied in the present Raman investigation. Energies of $\mathrm{Fe}^{2+}$ levels were obtained by numerical calculations, described in the text

tion and valence bands, spin-flip Raman scattering (SFRS) from donor-bound electrons represents the conduction-band splitting only. In addition, SFRS produces signatures narrower than those in magnetoreflectivity, ${ }^{22}$ allowing the $s-d$ exchange constant to be determined with higher precision in the former than in the latter. In this manner, $\alpha N_{0}$ was obtained in $\mathrm{Cd}_{1-x} \mathrm{Fe}_{x} \mathrm{Se}$ (Ref. 25) and in $\mathrm{Cd}_{1-x} \mathrm{Fe}_{x} \mathrm{~S} .{ }^{15}$ In addition, SFRS in these materials demonstrated the occurrence of a bound magnetic polaron in a Van Vleck system. ${ }^{15,16,25}$

In this paper we report results on $\mathrm{Cd}_{1-x} \mathrm{Fe}_{x} \mathrm{Te}$ obtained with resonant Raman scattering. Although similar Raman measurements were reported by Stühler et al.,${ }^{17}$ the present work includes SFRS and magnetization measurements on the same samples allowing the determination of $\alpha N_{0}$ and explores its anisotopy. We also emphasize that, in the absence of such magnetization measurements, Stühler et al. ${ }^{17} \mathrm{em}-$ ploying Raman scattering and polar magneto-optic Kerr effect (MOKE) could only determine the ratio $\alpha / \beta$. In addition, Raman transition of $\mathrm{Fe}^{2+}$ from its ground state to the first excited state and its Zeeman effect as a function of crystallographic orientation are reported and interpreted in the present paper.

\section{EXPERIMENT}

Raman scattering, wavelength-modulated reflectivity, and magnetization measurements were performed on (1 $\overline{1} 0)$
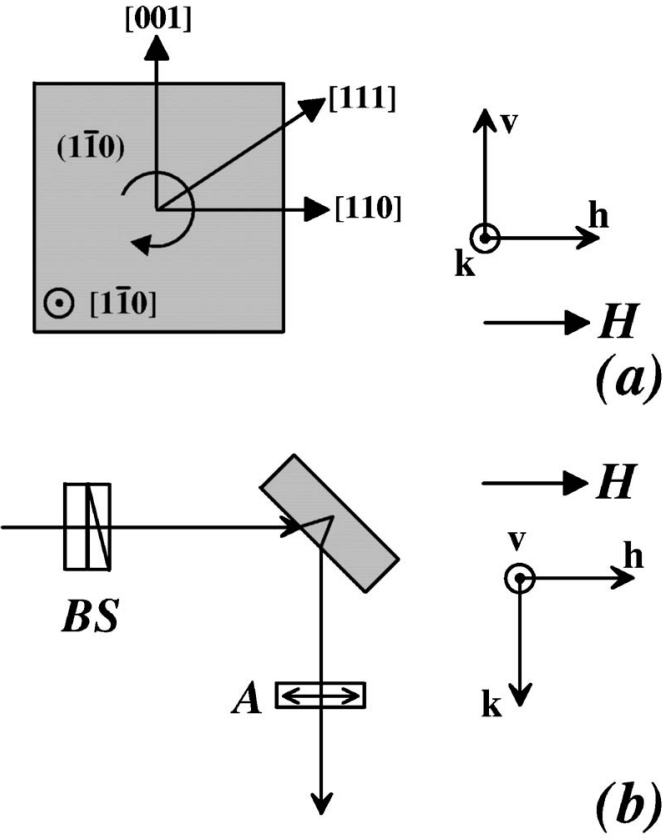

FIG. 2. (a) Back- and (b) $90^{\circ}$-scattering configurations for Raman measurements. The right-hand laboratory co-ordinate system $(\mathbf{k}, \mathbf{h}, \mathbf{v})$ is described in the text. $B S \equiv$ Babinet-Soleil compensator; $A \equiv$ linear analyzer.

samples cleaved from bulk crystals of $\mathrm{Cd}_{1-x} \mathrm{Fe}_{x} \mathrm{Te}$ grown by the modified vertical Bridgman method. The Raman line resulting from the $\Gamma_{1} \rightarrow \Gamma_{4}$ internal transition of $\mathrm{Fe}^{2+}$ was investigated with specimens of nominal concentration of $\mathrm{Fe}^{2+}$ in the range $10^{18}-10^{19}$ per $\mathrm{cm}^{3}$, since such samples yielded a Raman line narrower than those for much higher concentrations. SFRS and magnetization measurements were performed on samples with $x=0.009,0.012$, and 0.024 , as deduced from the energy of the free excitons..$^{20}$ The samples with $x=0.009$ and 0.012 were not oriented, whereas for the samples used to study the $\Gamma_{1} \rightarrow \Gamma_{4}$ transition, as well as for $x=0.024$, the [001], [110], and [111] directions were identified in the $(1 \overline{1} 0)$ plane with $x$ rays. Magnetization measurements were performed in the temperature range $2-300 \mathrm{~K}$ in a magnetic field with strengths up to $70 \mathrm{kOe}$, employing a Quantum Design MPMS XL7 superconducting quantum interference device (SQUID) magnetometer, the magnetic field being perpendicular to the cleaved surfaces. Wavelengthmodulated reflectivity spectra were obtained at low temperatures in zero magnetic field.

Raman spectra, excited by radiation from a tunable Ti:Sapphire laser, were recorded in the back- or $90^{\circ}$-scattering configurations schematically shown in Fig. 2. The Raman spectra were recorded under excitonic resonance enhancement with exciting radiation intensities not exceeding $2 \mathrm{~W} / \mathrm{cm}^{2}$ in order to minimize local heating effects. The scattered radiation was analyzed with a Spex double or, when a greater stray light rejection was desired, a triple spectrometer, and detected using standard photon-counting electronics. 


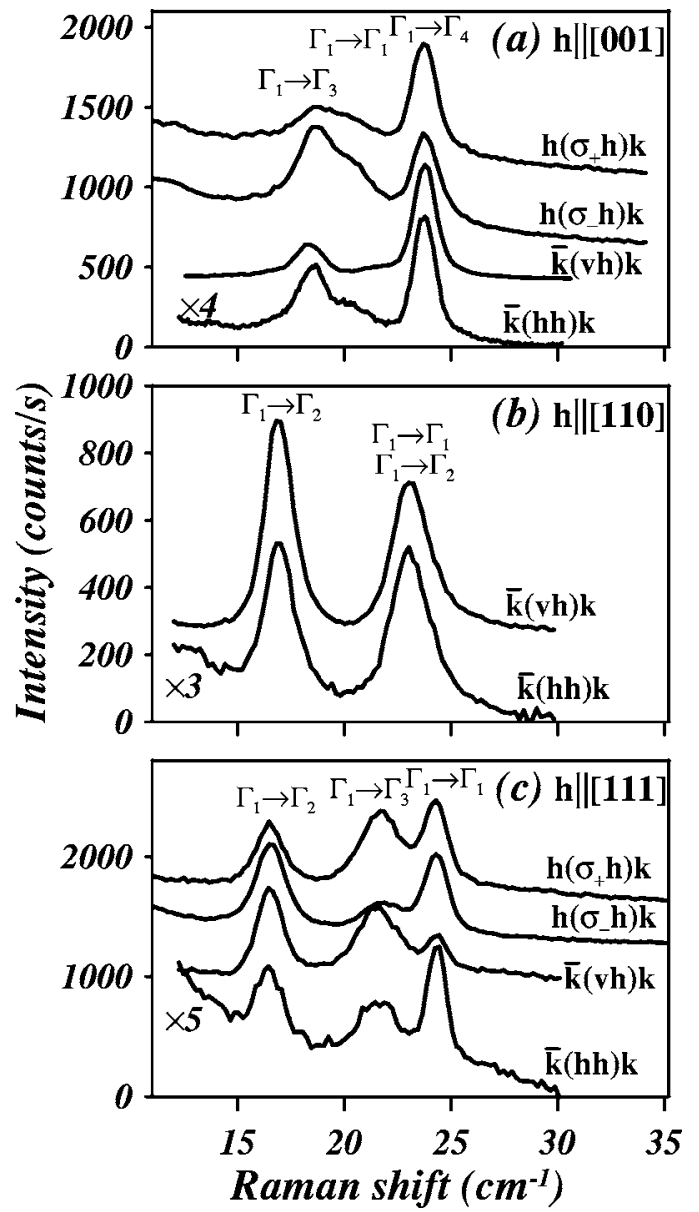

FIG. 3. Zeeman components of the $\Gamma_{1} \rightarrow \Gamma_{4}$ Raman transition of $\mathrm{Fe}^{2+}$, observed at $T=5 \mathrm{~K}$ and $B=60 \mathrm{kG}$ in different polarization configurations for magnetic field along (a) [100], (b) [110], or (c) [111]. Spectra are shifted vertically for clarity.

\section{RESULTS AND DISCUSSION}

\section{A. $\Gamma_{1} \rightarrow \Gamma_{4}$ transition of $\mathrm{Fe}^{2+}$}

Figures 3(a)-3(c) display the Zeeman components of the $\Gamma_{1} \rightarrow \Gamma_{4}$ Raman transition of $\mathrm{Fe}^{2+}$ recorded at $T=5 \mathrm{~K}$ and $B=60 \mathrm{kG}$. The measurements were performed on a sample with a cleaved $(1 \overline{1} 0)$ surface in the back- or $90^{\circ}$-scattering geometries. The backscattering geometry, including the direction of the applied magnetic field, is depicted in Fig. 2(a).

The direction of $\mathbf{H}$ and [1 $\overline{1} 0$ ] were fixed along $\mathbf{h}$ and $\mathbf{k}$ of the laboratory system, respectively. By rotating the sample around k, [110], [111], and [001] are successively brought into coincidence with $\mathbf{h}$. The Raman spectra were recorded in $\bar{k}(v h) k$ and $\bar{k}(h h) k$ polarization configurations, with incident radiation propagating along $\overline{\mathbf{k}}$ and polarized along $\mathbf{v}$ or $\mathbf{h}$ and backscattered along $\mathbf{k}$ and analyzed along $\mathbf{h}$. For the $90^{\circ}$-scattering configuration [Fig. 2(b)], the sample with its [001] or [110] along $\mathbf{v}$ is rotated around $\mathbf{v}$ until [010] or [1]1] , respectively, is brought into coincidence with $\mathbf{h}$. The incident light with either $\sigma_{-}$or $\sigma_{+}$polarization propagates along $\mathbf{h}$, and the $\mathbf{h}$-polarized scattered light along $\mathbf{k}$, resulting in $h\left(\sigma_{-} h\right) k$ or $h\left(\sigma_{+} h\right) k$ polarization configurations, respec-
TABLE I. Selection rules for Raman transitions of $\mathrm{Fe}^{2+}$ in the presence of an external magnetic field along $\mathbf{h} \|[001]$.

\begin{tabular}{lcccc}
\hline \hline & $\bar{k}(v h) k$ & $\bar{k}(h h) k$ & $h\left(\sigma_{-}, h\right) k$ & $h\left(\sigma_{+}, h\right) k$ \\
\hline$\Gamma_{1} \rightarrow \Gamma_{1}$ & forbidden & allowed & forbidden & forbidden \\
$\Gamma_{1} \rightarrow \Gamma_{3}$ & allowed & forbidden & allowed & forbidden \\
$\Gamma_{1} \rightarrow \Gamma_{4}$ & allowed & forbidden & forbidden & allowed \\
\hline \hline
\end{tabular}

tively. We note the above orientation of the sample with respect to the incident and scattered light is unavoidable in view of the small scattering volume probed under resonance conditions. Consequently, the incident, say, $\sigma_{+}$polarization outside the sample gets somewhat "contaminated" with the $\sigma_{-}$and $h$ polarizations upon entering the sample. Similarly, the scattered light analyzed along $\mathbf{h}$ outside the sample has additional contributions with $\sigma_{ \pm}$polarizations emerging from the sample. Thus the chosen geometry results in a departure from the exact $90^{\circ}$-scattering expected had the crystal been transparent.

Raman tensors, characterizing the observed transitions, can be obtained using group theory. In the following, Raman tensors are given in the $\mathbf{v}, \mathbf{k}$, and $\mathbf{h}$ right-hand coordinate system of Fig. 2(b). For $\mathbf{h} \|[001]$, the presence of the magnetic field reduces the $T_{d}$ site symmetry of $\mathrm{Fe}^{2+}$ to $S_{4}$, with the following compatibility between corresponding representations: $\Gamma_{1}\left(T_{d}\right) \Rightarrow \Gamma_{1}\left(S_{4}\right), \quad \Gamma_{4}\left(T_{d}\right) \Rightarrow \Gamma_{1}+\Gamma_{3}+\Gamma_{4}\left(S_{4}\right)$. Raman tensors for transitions $\Gamma_{1} \rightarrow \Gamma_{1}, \Gamma_{1} \rightarrow \Gamma_{3}$, and $\Gamma_{1} \rightarrow \Gamma_{4}$ belong to representations $\Gamma_{1}, \Gamma_{3}$, and $\Gamma_{4}$ of group $S_{4}$, respectively, and are given by

$$
\begin{aligned}
& \alpha\left(\Gamma_{1}\right)=\left(\begin{array}{ccc}
a_{11} & a_{12}^{\prime} & 0 \\
-a_{12}^{\prime} & a_{11} & 0 \\
0 & 0 & a_{33}
\end{array}\right) \\
& \alpha\left(\Gamma_{3}\right)=\left(\begin{array}{ccc}
0 & 0 & c+c^{\prime} \\
0 & 0 & i\left(c+c^{\prime}\right) \\
c-c^{\prime} & i\left(c-c^{\prime}\right) & 0
\end{array}\right) \text {; } \\
& \alpha\left(\Gamma_{4}\right)=\left(\begin{array}{ccc}
0 & 0 & \left(c+c^{\prime}\right)^{*} \\
0 & 0 & -i\left(c+c^{\prime}\right)^{*} \\
\left(c-c^{\prime}\right)^{*} & -i\left(c-c^{\prime}\right)^{*} & 0
\end{array}\right) .
\end{aligned}
$$

Selection rules arising from Eqs. (1) for the back- and $90^{\circ}$ scattering are summarized in Table I.

The Raman spectra for $\mathbf{h} \|[001]$ [Fig. 3(a)] contain three peaks in $h\left(\sigma_{-}, h\right) k, h\left(\sigma_{+}, h\right) k$, and $\bar{k}(h h) k$, and two in $\bar{k}(v h) k$. Their labeling shown in Fig. 3(a) follows from the selection rules in Table I together with (i) the stronger $\Gamma_{1} \rightarrow \Gamma_{3}$ peak observed in $h\left(\sigma_{-}, h\right) k$ than in $h\left(\sigma_{+}, h\right) k$, (ii) the stronger $\Gamma_{1} \rightarrow \Gamma_{4}$ peak in $h\left(\sigma_{+}, h\right) k$ than in $h\left(\sigma_{-}, h\right) k$, and (iii) presence of $\Gamma_{1} \rightarrow \Gamma_{1}$ in $\bar{k}(h h) k$ and its absence in $\bar{k}(v h) k$. We attribute the appearance of $\Gamma_{1} \rightarrow \Gamma_{3}$ in $h\left(\sigma_{+}, h\right) k$ and that of $\Gamma_{1} \rightarrow \Gamma_{4}$ in $h\left(\sigma_{-}, h\right) k$ to the departure from the exact rightangle scattering mentioned above. For the same reason the $\Gamma_{1} \rightarrow \Gamma_{1}$ transition is seen as a shoulder in the forbidden 
TABLE II. Selection rules for Raman transitions of $\mathrm{Fe}^{2+}$ in the presence of an external magnetic field along $\mathbf{h} \|[110]$.

\begin{tabular}{lcc}
\hline \hline & $\bar{k}(v h) k$ & $\bar{k}(h h) k$ \\
\hline$\Gamma_{1} \rightarrow \Gamma_{1}$ & forbidden & allowed \\
$\Gamma_{1} \rightarrow \Gamma_{2}$ & allowed & forbidden \\
\hline \hline
\end{tabular}

$h\left(\sigma_{-}, h\right) k$ and $h\left(\sigma_{+}, h\right) k$. The appearance of $\Gamma_{1} \rightarrow \Gamma_{3}$ and $\Gamma_{1} \rightarrow \Gamma_{4}$ with distinctly lower intensity in the forbidden $\bar{k}(h h) k$ configuration than in $\bar{k}(v h) k$ is attributed to the leakage of the lines, allowed in $\bar{k}(h v) k$, through the imperfect analyzer. While the stronger intensity of $\Gamma_{1} \rightarrow \Gamma_{1}$ in $h\left(\sigma_{ \pm}, h\right) k$ than in $\bar{k}(h h) k$ is not completely understood, we note that a comparison of intensities in $h\left(\sigma_{ \pm}, h\right) k$ and $\bar{k}(h h) k$ involves spectra recorded in two separate experiments. For a strict comparison between spectra obtained in separate configurations, e.g., $90^{\circ}$ - and backscattering, one has to ensure that the scattering volumes are identical in the two experiments, given the inhomogeneity of $\mathrm{Fe}^{2+}$, especially at low concentrations as in our samples. A change in the excitonic resonance condition exploited in our experiments on $\Gamma_{1} \rightarrow \Gamma_{1}$ in $h\left(\sigma_{ \pm}, h\right) k$ with respect to that in $\bar{k}(h h) k$ could well account for its observed relative intensities. The relative positions of the identified transitions are consistent with the order of the excited levels with increasing energy being $\Gamma_{3}, \Gamma_{1}, \Gamma_{4}$, as determined in the present investigation from a numerical calculations of energies and eigenfunctions of $\mathrm{Fe}^{2+}$, described later in the text.

For $\mathbf{h} \|[110]$ the site symmetry of $\mathrm{Fe}^{2+}$ is reduced from $T_{d}$ to $C_{s}$, the compatibility relations being $\Gamma_{1}\left(T_{d}\right) \Rightarrow \Gamma_{1}\left(C_{s}\right)$, $\Gamma_{4}\left(T_{d}\right) \Rightarrow \Gamma_{1}+2 \Gamma_{2}\left(C_{s}\right)$. The order of the excited levels is $\Gamma_{2}$, $\Gamma_{1}, \Gamma_{2}$ below and $\Gamma_{2}, \Gamma_{2}, \Gamma_{1}$ above $40 \mathrm{kG}$ according to the numerical calculations, showing that $\Gamma_{1}$ and the upper $\Gamma_{2}$ cross around that magnetic field. Raman tensors for $\Gamma_{1} \rightarrow \Gamma_{1}$ and $\Gamma_{1} \rightarrow \Gamma_{2}$ in this symmetry belong to $\Gamma_{1}$ and $\Gamma_{2}$ of $C_{s}$, respectively, with

$$
\begin{gathered}
\alpha\left(\Gamma_{1}\right)=\left(\begin{array}{ccc}
a_{11} & a_{12}+a_{12}^{\prime} & 0 \\
a_{12}-a_{12}^{\prime} & a_{22} & 0 \\
0 & 0 & a_{33}
\end{array}\right), \\
\alpha\left(\Gamma_{2}\right)=\left(\begin{array}{ccc}
0 & 0 & c_{13}+c_{13}^{\prime} \\
0 & 0 & c_{23}+c_{23}^{\prime} \\
c_{13}-c_{13}^{\prime} & c_{23}-c_{23}^{\prime} & 0
\end{array}\right),
\end{gathered}
$$

yielding the selection rules shown in Table II.

In accordance with these selection rules, two $\Gamma_{1} \rightarrow \Gamma_{2}$ Raman transitions are observed in $\bar{k}(v h) k$ [Fig. 3(b)]. In $\bar{k}(h h) k$, two lines with the same Raman shifts as those of the $\Gamma_{1} \rightarrow \Gamma_{2}$ transitions in $\bar{k}(v h) k$ can be seen, implying that their appearance is related to the leakage of the $\Gamma_{1} \rightarrow \Gamma_{2}$ lines, allowed in $\bar{k}(h v) k$, through the imperfect analyzer. However, the change in relative intensities of the Raman lines when going from $\bar{k}(v h) k$ to $\bar{k}(h h) k$ [Fig. 3(b)] suggests an intensity
TABLE III. Selection rules for Raman transitions of $\mathrm{Fe}^{2+}$ in the presence of an external magnetic field along $\mathbf{h} \|[111]$.

\begin{tabular}{lcccc}
\hline \hline & $\bar{k}(v h) k$ & $\bar{k}(h h) k$ & $h\left(\sigma_{-}, h\right) k$ & $h\left(\sigma_{+}, h\right) k$ \\
\hline$\Gamma_{1} \rightarrow \Gamma_{1}$ & forbidden & allowed & forbidden & forbidden \\
$\Gamma_{1} \rightarrow \Gamma_{2}$ & allowed & forbidden & allowed & forbidden \\
$\Gamma_{1} \rightarrow \Gamma_{3}$ & allowed & forbidden & forbidden & allowed \\
\hline \hline
\end{tabular}

contribution to the higher-energy line, present in $\bar{k}(h h) k$ and absent in $\bar{k}(v h) k$. Indeed, our numerical calculations yielded very close Raman shifts for $\Gamma_{1} \rightarrow \Gamma_{1}$ and the higher-energy $\Gamma_{1} \rightarrow \Gamma_{2}$. It thus appears that $\Gamma_{1} \rightarrow \Gamma_{1}$ merges with the higherenergy $\Gamma_{1} \rightarrow \Gamma_{2}$ in $\bar{k}(h h) k$.

In the case of $\mathbf{h} \|[111], T_{d}$ reduces to $C_{3}$, generating the compatibilities $\Gamma_{1}\left(T_{d}\right) \Rightarrow \Gamma_{1}\left(C_{3}\right), \quad \Gamma_{4}\left(\mathrm{~T}_{d}\right) \Rightarrow \Gamma_{1}+\Gamma_{2}+\Gamma_{3}\left(C_{3}\right)$. Accordingly, the Raman tensors for $\Gamma_{1} \rightarrow \Gamma_{1}, \Gamma_{1} \rightarrow \Gamma_{2}$, and $\Gamma_{1} \rightarrow \Gamma_{3}$ are given by

$$
\begin{aligned}
& \alpha\left(\Gamma_{1}\right)=\left(\begin{array}{ccc}
a_{11} & a_{12}^{\prime} & 0 \\
-a_{12}^{\prime} & a_{11} & 0 \\
0 & 0 & a_{33}
\end{array}\right) \\
& \alpha\left(\Gamma_{2}\right)=\left(\begin{array}{ccc}
c_{1} & -i c_{1} & c_{2}+c_{2}^{\prime} \\
-i c_{1} & -c_{1} & i\left(c_{2}+c_{2}^{\prime}\right) \\
c_{2}-c_{2}^{\prime} & i\left(c_{2}-c_{2}^{\prime}\right) & 0
\end{array}\right) \\
& \alpha\left(\Gamma_{3}\right)=\left(\begin{array}{ccc}
c_{1} & i c_{1} & \left(c_{2}+c_{2}^{\prime}\right)^{*} \\
i c_{1} & -c_{1} & -i\left(c_{2}+c_{2}^{\prime}\right)^{*} \\
\left(c_{2}-c_{2}^{\prime}\right)^{*} & -i\left(c_{2}-c_{2}^{\prime}\right)^{*} & 0
\end{array}\right) .
\end{aligned}
$$

Selection rules, deduced from Eqs. (3), are given in Table III.

Raman spectra recorded at $60 \mathrm{kG}$ [Fig. 3(c)] exhibit three peaks in all four scattering configurations. Their identification on the basis of the selection rules of Table III is possible due to (i) the $\Gamma_{1} \rightarrow \Gamma_{2}$ peak being stronger in $h\left(\sigma_{-}, h\right) k$ than in $h\left(\sigma_{+}, h\right) k$, (ii) $\Gamma_{1} \rightarrow \Gamma_{3}$ being stronger in $h\left(\sigma_{+}, h\right) k$ than in $h\left(\sigma_{-}, h\right) k$, and (iii) the relative intensity of $\Gamma_{1} \rightarrow \Gamma_{1}$ with respect to those of $\Gamma_{1} \rightarrow \Gamma_{2}$ and $\Gamma_{1} \rightarrow \Gamma_{3}$ in $\bar{k}(v h) k$ and $\bar{k}(h h) k$. The appearance of $\Gamma_{1} \rightarrow \Gamma_{2}$ in $h\left(\sigma_{+}, h\right) k$ and that of $\Gamma_{1} \rightarrow \Gamma_{3}$ in $h\left(\sigma_{-}, h\right) k$, as well as the appearance of $\Gamma_{1} \rightarrow \Gamma_{1}$ in $h\left(\sigma_{ \pm}, h\right) k$, are related to the departures from the exact $90^{\circ}$ scattering. While observation of $\Gamma_{1} \rightarrow \Gamma_{2}$ and $\Gamma_{1} \rightarrow \Gamma_{3}$ in $\bar{k}(h h) k$ must be attributed to the leakage of the lines, excited in $\bar{k}(h v) k$, through the imperfect analyzer, that of $\Gamma_{1} \rightarrow \Gamma_{1}$ in $\bar{k}(v h) k$ as well as its stronger intensity in $h\left(\sigma_{ \pm}, h\right) k$ than in $\bar{k}(h h) k$ are not fully understood. Raman shifts of the identified transitions are consistent with the order of the excited levels, determined from the calculations, being $\Gamma_{2}, \Gamma_{1}, \Gamma_{3}$ below $27 \mathrm{kG}$, and $\Gamma_{2}, \Gamma_{3}, \Gamma_{1}$ above.

In order to interpret the observed Raman spectra quantitatively we calculated numerically energies and eigenfunctions of $\mathrm{Fe}^{2+}$, and the corresponding Raman shifts as functions of the magnetic field, following the procedure in Ref. 
14. The Hamiltonian for $\mathrm{Fe}^{2+}$ in $\mathrm{CdTe}$ in the presence of a magnetic induction $\mathbf{B}$,

$$
\mathcal{H}=\mathcal{H}_{0}+\mathcal{H}_{C F}+\mathcal{H}_{S O}+\mathcal{H}_{Z}
$$

includes $\mathcal{H}_{0}$, the Hamiltonian of the free $\mathrm{Fe}^{2+}, \mathcal{H}_{S O}=\lambda \mathbf{L} \cdot \mathbf{S}$, the spin-orbit interaction, $\mathcal{H}_{Z}=\mu_{B} \mathbf{B} \cdot(\mathbf{L}+2 \mathbf{S})$, the Zeeman interaction, with $\mu_{B}$ being the Bohr magneton, and the crystalfield Hamiltonian $\mathcal{H}_{C F}$, defined in terms of $\Delta$. The manifold of the 25 wave functions of the degenerate ground state $(S$ $=2, L=2)$ of the free $\mathrm{Fe}^{2+}$ is chosen as a complete set and $\mathcal{H}_{C F}+\mathcal{H}_{S O}+\mathcal{H}_{Z}$ is treated together as a perturbation, with $\mathcal{H}_{C F}$ expressed in terms of operator equivalents. ${ }^{26} \mathrm{~A}$ $25 \times 25$ matrix for $\mathcal{H}_{C F}+\mathcal{H}_{S O}+\mathcal{H}_{Z}$ is formed, and the corresponding secular equation is solved numerically to yield energies and eigenfunctions for the 25 lowest levels of $\mathrm{Fe}^{2+}$ in CdTe. Numerical calculations using $\Delta=2480 \mathrm{~cm}^{-1}$ and $\lambda=-100 \mathrm{~cm}^{-1}$ reproduce the zero-field Raman shift of $\Gamma_{1}\left(T_{d}\right) \rightarrow \Gamma_{4}\left(T_{d}\right)$ and an energy of a $\Gamma_{1}\left(T_{d}\right) \rightarrow \Gamma_{5}\left(T_{d}\right)$ infrared transition of $\mathrm{Fe}^{2+}$ observed at $2282.8 \mathrm{~cm}^{-1}$ in CdTe by Udo et al. ${ }^{27}$ The calculated eigenfunctions can be assigned to representations of $S_{4}, C_{s}$, or $C_{3}$ by checking their symmetry properties under operations of the corresponding group.

The Raman shifts determined from the above calculations are compared to experimental values in Fig. 4. While the calculations reproduce experimental Zeeman splittings reasonably well, the observed small difference (a maximum of $1.5 \mathrm{~cm}^{-1}$ at the highest fields) may have its origin in a dynamic Jahn-Teller effect ${ }^{9}$ modifying the energy levels of $\mathrm{Fe}^{2+}$.

\section{B. Electron SFRS}

In this section, the focus is on the $s-d$ exchange interaction in $\mathrm{Cd}_{1-x} \mathrm{Fe}_{x} \mathrm{Te}$ as manifested in the electron SFRS from samples with $x$ sufficient to result in a ternary alloy. The Fe concentration was determined from the energy of the free excitonic signature $\left[E_{g x}(x)\right]$ observed in wavelengthmodulated reflectivity at zero magnetic field, in conjunction with the calibration $E_{g x}(x)=1.594+1.78 x(\mathrm{eV})$ obtained by Testelin et al. ${ }^{20}$

Figure 5 shows electron SFRS spectra recorded for $x=0.024$ at $2 \mathrm{~K}$. The Raman shift of electron SFRS, equal to the Zeeman splitting of the conduction-band minimum, is given by $\mathrm{y}^{7,28}$

$$
\hbar \omega_{S F R S}=\Delta_{C B}=g^{*} \mu_{B} B-x \alpha N_{0}\left\langle\left\langle S_{z}\right\rangle\right\rangle,
$$

the first term describing the intrinsic Zeeman splitting in the CdTe host, characterized by $g^{*}=-1.676,{ }^{7}$ and the second term, known as the $s$ - $d$ exchange energy, arising from $s$ - $d$ exchange interaction. In this expression, $\alpha N_{0}$ is the $s-d$ exchange constant, and $\left\langle\left\langle S_{z}\right\rangle\right\rangle$ a thermal and spatial average of the magnetic ion spin projection on the direction of magnetic field. Noting the expression for macroscopic magnetization $M_{m}^{*}$ associated with the TMI is given by

$$
M_{m}^{*}=-\frac{\mu_{B} N_{A} x}{W(x)}\left\langle\left\langle L_{z}+2 S_{z}\right\rangle\right\rangle,
$$

where $W(x)$ is the molecular weight of the DMS, the electron spin-flip Raman shift can be rewritten as

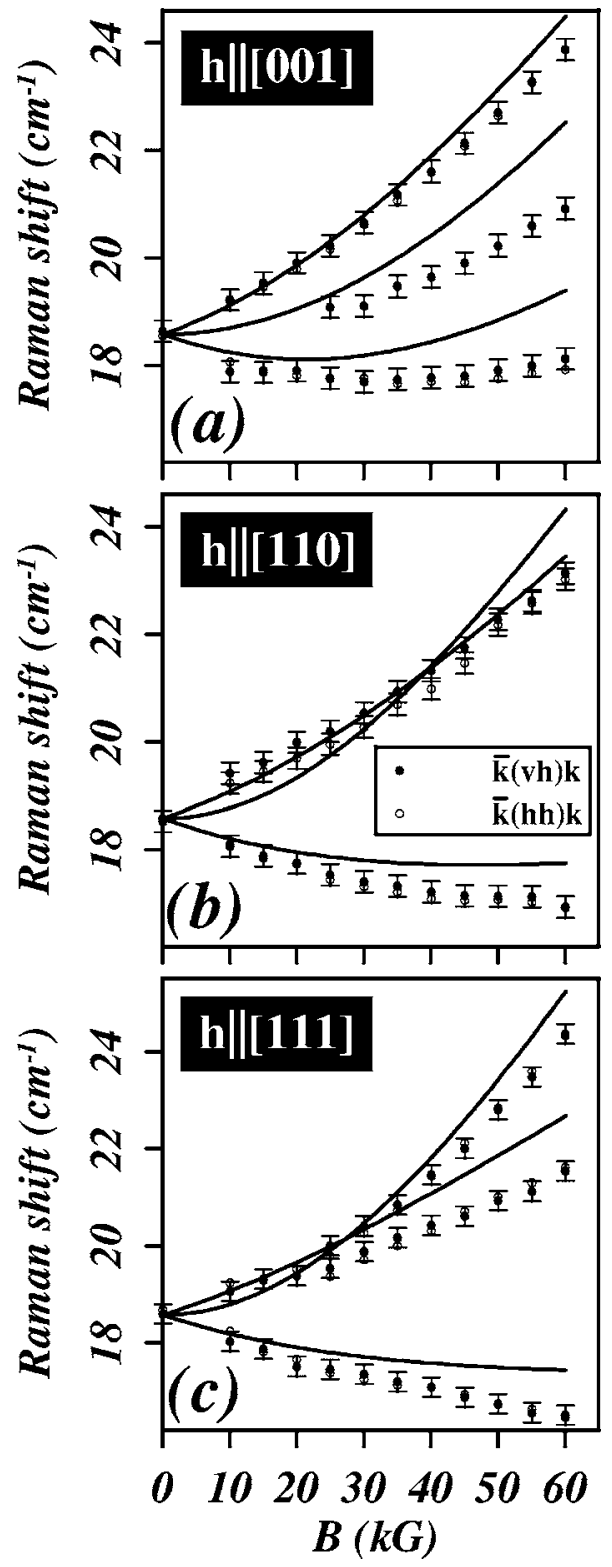

FIG. 4. Raman shifts of the Zeeman components of $\Gamma_{1} \rightarrow \Gamma_{4}$ as functions of $\mathbf{B}$ for $\mathbf{h}$ along (a) [100], (b) [110], or (c) [111]: dots and crosses: experimental data recorded in $\bar{k}(v h) k$ and $\bar{k}(h h) k$ configurations, respectively; solid lines: numerically calculated shifts, using $\Delta=2480 \mathrm{~cm}^{-1}$ and $\lambda=-100 \mathrm{~cm}^{-1}$.

$$
\hbar \omega_{S F R S}=g^{*} \mu_{B} B+\alpha N_{0}\left(\frac{W(x)}{\mu_{B} N_{A}}\right)\left(\frac{\left\langle\left\langle S_{z}\right\rangle\right\rangle}{\left\langle\left\langle L_{z}+2 S_{z}\right\rangle\right\rangle}\right) M_{m}^{*} .
$$

The quantity $\left\langle L_{z}+2 S_{z}\right\rangle /\left\langle S_{z}\right\rangle$ for an isolated $\mathrm{Fe}^{2+}$ in $\mathrm{Cd}_{1-x} \mathrm{Fe}_{x} \mathrm{Te}$ at $1.8 \mathrm{~K}$ was theoretically calculated ${ }^{20}$ to be $2.29 \pm 0.01$, with a negligible dependence on the magneticfield orientation and for $B \leqslant 70 \mathrm{kG}$.

Subtracting the intrinsic contribution from the SFRS shift, one obtains the $s-d$ exchange energy. In Fig. 6 this exchange energy for $\mathrm{Cd}_{0.988} \mathrm{Fe}_{0.012} \mathrm{Te}$ is plotted as a function of magnetic field at several temperatures. The plots clearly display features characteristic of Van Vleck magnetization: absence of saturation even at high fields and lowest temperatures, 


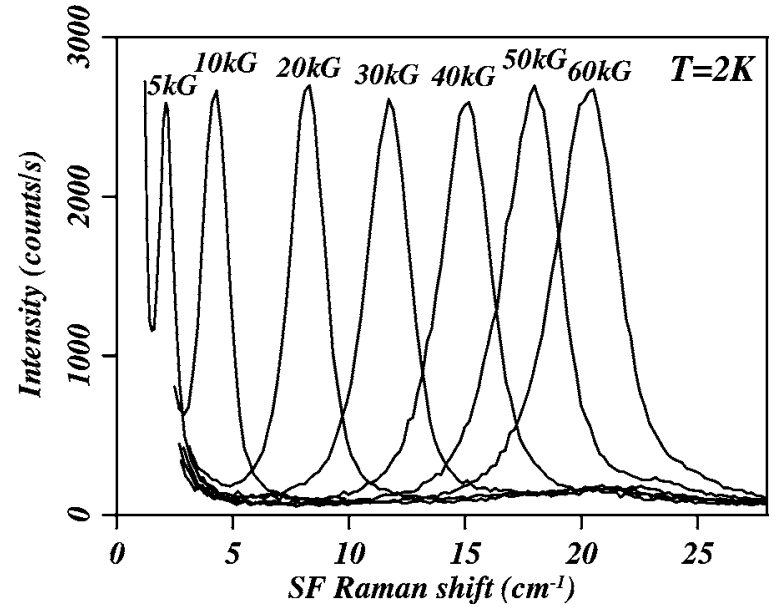

FIG. 5. Electron spin-flip Raman scattering (SFRS) spectra of $\mathrm{Cd}_{0.976} \mathrm{Fe}_{0.024} \mathrm{Te}$ at $T=2 \mathrm{~K}$ recorded at different B's.

and a weak temperature dependence at low temperatures, below $5 \mathrm{~K}$ in the present case.

The unusual behavior of the half width of the SFRS line as displayed in Fig. 5 is noteworthy. After taking due account of the instrumental broadening, the width of SFRS line is found to change linearly with the $s-d$ exchange energy. In contrast, our study of SFRS in $\mathrm{Cd}_{1-x} \mathrm{Mn}_{x} \mathrm{Te}$ revealed a significantly broader Raman line, whose width first decreases with increasing $s-d$ exchange energy, and then increases again (Fig. 7). This behavior is similar to that observed in $\mathrm{Cd}_{1-x} \mathrm{Fe}_{x} \mathrm{Se}$ and $\mathrm{Cd}_{1-x} \mathrm{Mn}_{x} \mathrm{Se}$ by Heiman et al. ${ }^{25}$ who have shown that the SFRS line width in a DMS is affected by two factors: (i) compositional alloy fluctuations and (ii) stochastic thermal fluctuations of the TMI magnetic moment. While both contribute to the linewidth in $\mathrm{Cd}_{1-x} \mathrm{Mn}_{x} \mathrm{Se}^{25}$ and

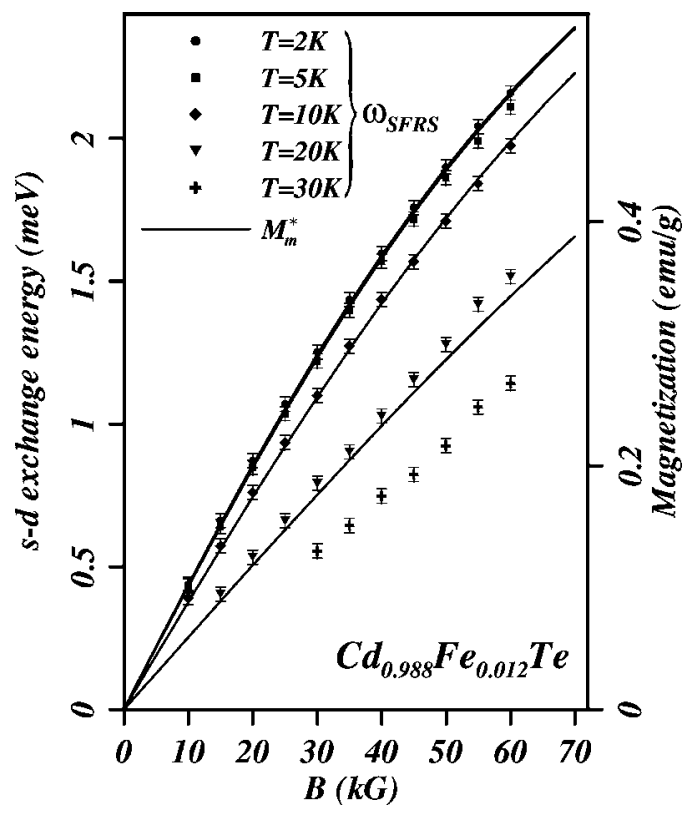

FIG. 6. $s$ - $d$ exchange energy of $\mathrm{Cd}_{0.988} \mathrm{Fe}_{0.012} \mathrm{Te}$, deduced from electron SFRS, as a function of $\mathbf{B}$ at several temperatures. Solid lines are magnetization (corrected for the host diamagnetism) of the same sample as a function of $\mathbf{B}$ at $T=2,5,10$, and $20 \mathrm{~K}$.

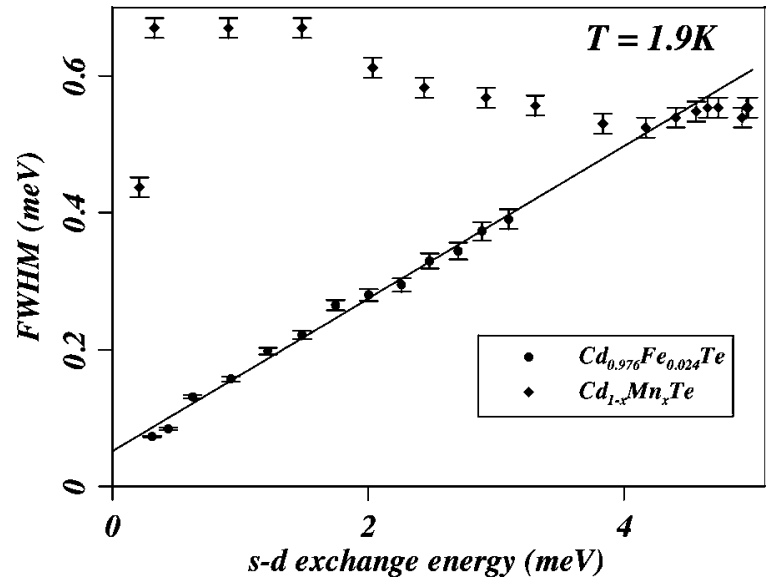

FIG. 7. Full width at half maximum of SFRS line as a function of the $s-d$ exchange energy in $\mathrm{Cd}_{0.988} \mathrm{Fe}_{0.012} \mathrm{Te}$ and $\mathrm{Cd}_{1-x} \mathrm{Mn}_{x} \mathrm{Te}$.

$\mathrm{Cd}_{1-x} \mathrm{Mn}_{x} \mathrm{Te}$ (present study), the latter is absent in $\mathrm{Cd}_{1-x} \mathrm{Fe}_{x} \mathrm{Se}$ (Ref. 25) and $\mathrm{Cd}_{1-x} \mathrm{Fe}_{x} \mathrm{Te}$ (present study). This difference can be traced to the ground state of $\mathrm{Fe}^{2+}$ being a singlet; hence the SFRS linewidth, affected only by the compositional alloy fluctuations in the Fe-based DMSs, increases linearly with the $s-d$ exchange energy. We mention that such a linear increase in the linewidth with the $s-d$ exchange energy is expected for Mn-based DMSs at higher magnetic fields, where effects of thermal fluctuations become negligible.

Anisotropy of $M_{m}^{*}$ reported in magnetization measurements of $\mathrm{Cd}_{1-x} \mathrm{Fe}_{x} \mathrm{Te}$ (Refs. 14 and 29) can also be demonstrated for the $s-d$ exchange energy using SFRS (see Fig. 8). Our SFRS measurements at fixed $\mathbf{B}$ and $T$, made on different spots on the surface of the same $\mathrm{Cd}_{1-x} \mathrm{Fe}_{x} \mathrm{Te}$, revealed $\sim 6 \%$ inhomogeniety in $\mathrm{Fe}^{2+}$ distribution. Thus special measures were taken to ensure that the laser beam was focused at the same spot of the sample surface for different directions of magnetic field. This was achieved by attaching a $500-\mu \mathrm{m}$-diameter aluminum diaphragm to the sample surface. The observed anisotropy of the $s-d$ exchange energy is consistent with that observed in magnetization ${ }^{14,29}$ On the basis of Eq. (7), this indicates that the $s-d$ exchange constant $\alpha N_{0}$ is isotropic.

In Fig. 9, the $s-d$ exchange energy is plotted vs magnetization for the three $\mathrm{Cd}_{1-x} \mathrm{Fe}_{x} \mathrm{Te}$ samples investigated. The data for each sample can be fitted by a linear dependence, as expected from Eq. (7). (In these measurements, we neglected the anisotropy of magnetization, since the inhomogeniety effects are stronger than the anisotropy ${ }^{14}$ and cannot be avoided in magnetization measurements.) The slope of the fits clearly decreases with increasing $\mathrm{Fe}$ concentration. This can be explained by an increasing effect of antiferromagnetic interactions ${ }^{29}$ of the nearest-neighbor $(\mathrm{NN}) \mathrm{Fe}^{2+}-\mathrm{Fe}^{2+}$ pairs, as well as those between more distant ions, resulting in a decrease of $\left\langle\left\langle S_{z}\right\rangle\right\rangle /\left\langle\left\langle L_{z}+2 S_{z}\right\rangle\right\rangle$ compared to $\left\langle S_{z}\right\rangle /\left\langle L_{z}+2 S_{z}\right\rangle$ of an isolated $\mathrm{Fe}^{2+}$. In the simplest approach, we neglect the $\mathrm{Fe}-\mathrm{Fe}$ interactions beyond $\mathrm{NN}$ because of the short-range $d-d$ interaction and assume the spin component of NN to be zero because of low magnetic fields $(\leqslant 60 \mathrm{kOe})$ employed (see Fig. 8 in Ref. 29); we also neglect contributions from 


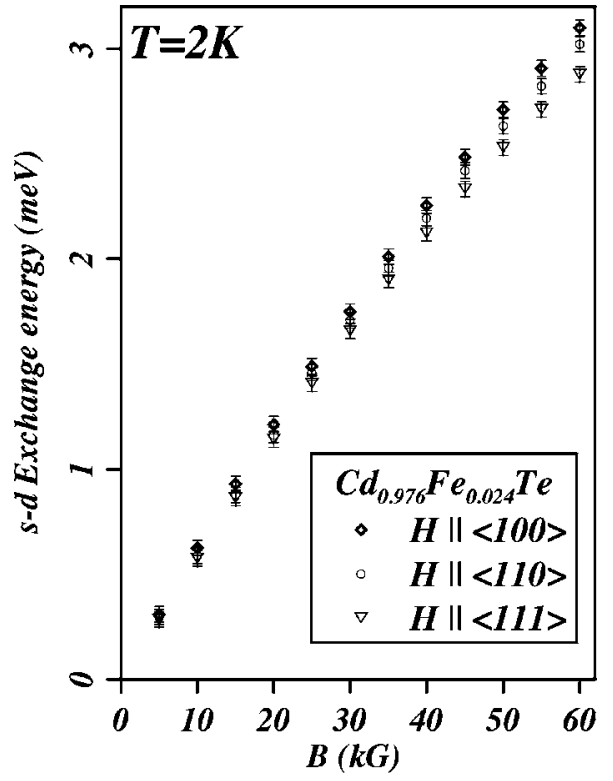

FIG. 8. Anisotropy of $s$ - $d$ exchange energy in $\mathrm{Cd}_{0.976} \mathrm{Fe}_{0.024} \mathrm{Te}$ revealed by electron SFRS.

NN triplets, quadruplets, etc., because of their low probability of occurrence, following Testelin et al. ${ }^{29}$ Thus NN pairs contribute to magnetization only via orbital angular momentum, while the rest (isolated+other than NN) contribute via both orbital and spin parts as isolated $\mathrm{Fe}^{2+}$ ions. With $P_{2}(x)=12 x(1-x)^{18}$ (Ref. 29) being the probability that $\mathrm{Fe}^{2+}$ ion belongs to an isolated $\mathrm{NN}$ pair,

$$
\frac{\left\langle\left\langle L_{z}+2 S_{z}\right\rangle\right\rangle}{\left\langle\left\langle S_{z}\right\rangle\right\rangle}=\frac{P_{2}(x)\left\langle L_{z}\right\rangle^{N N}+\left[1-P_{2}(x)\right]\left\langle L_{z}+2 S_{z}\right\rangle^{i i}}{\left[1-P_{2}(x)\right]\left\langle S_{z}\right\rangle^{i i}},
$$

where the supersctipt "ii" stands for isolated ions. Since $\left\langle L_{z}+2 S_{z}\right\rangle^{i i} /\left\langle S_{z}\right\rangle^{i i}=2.29 \quad$ at $1.8 \mathrm{~K}$, then $\left\langle L_{z}\right\rangle^{N N}=\left\langle L_{z}\right\rangle^{i i}$ $=0.29\left\langle S_{z}\right\rangle^{i i}$. Using Eq. (8), we calculated $\left\langle\left\langle L_{z}+2 S_{z}\right\rangle\right\rangle /\left\langle\left\langle S_{z}\right\rangle\right\rangle$, shown in Table IV together with $W(x)$, for the three samples studied. Finally, from the slopes of the fits in Fig. 9, $\alpha N_{0}$ was determined for the three samples. The observed reduction of $\alpha N_{0}$ with the increasing Fe concentration can be attributed to the antiferromagnetic exchange interactions ${ }^{29}$ between distant (beyond NN) Fe-Fe pairs, neglected in our simple analysis. Since these effects become less important at lower $\mathrm{Fe}$ concentrations, we choose $\alpha N_{0}=244 \pm 10 \mathrm{meV}$, obtained for the lowest $x$ studied. The $s$ - $d$ exchange constant thus deduced is in agreement with that determined from the Zeeman effect of the excitonic signature observed in modulated reflectivity. ${ }^{22}$

\section{CONCLUDING REMARKS}

Among the II-VI DMSs based on $3 d$ TMIs, those with $\mathrm{Fe}^{2+}$ are unique by virtue of the Van Vleck paramagnetism they display. The Zeeman effect of the $\Gamma_{1} \rightarrow \Gamma_{4}$ electronic Raman line is satisfactorily explained on the basis of crystal field, spin-orbit, and Zeeman interactions. This implies the relatively small effect of dynamic Jahn-Teller interaction in the levels originating from the ${ }^{5} \Gamma_{3}$ orbital doublet.

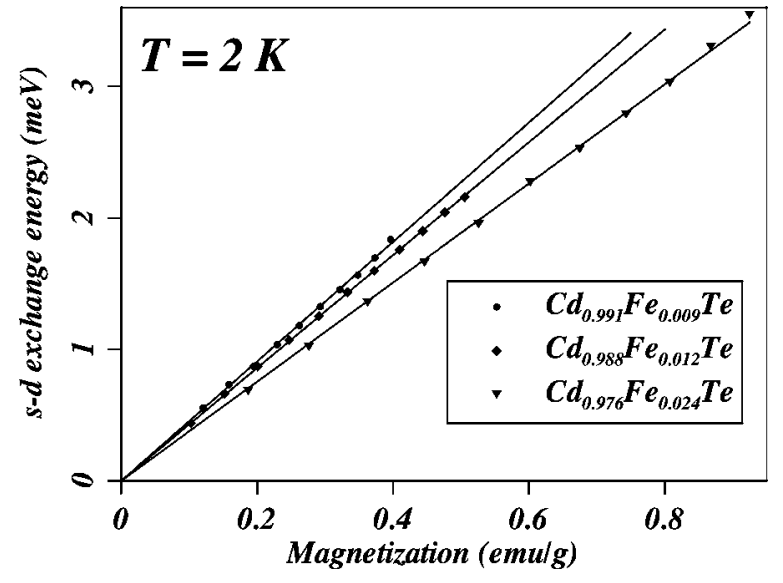

FIG. 9. $s-d$ exchange energy vs magnetization, determined for three $\mathrm{Cd}_{1-x} \mathrm{Fe}_{x}$ Te samples at $2 \mathrm{~K}$ with $\mathbf{B}$ in the range $10-60 \mathrm{kG}$.

The observed anisotropy of the Zeeman effect underlies that of the corresponding macroscopic magnetization and, in turn through $s-d$ interaction, is manifested in the spin-flip Raman shifts of donor-bound electrons. The versatility of SFRS is emphasized in the current investigation. On the one hand, the Raman shift of SFRS clearly exhibits all the characteristic features of the Van Vleck magnetization, such as absence of saturation at lowest temperature and highest magnetic field, a weak temperature dependence below $5 \mathrm{~K}$, and the anisotropy of the magnetization. On the other hand, it also characterizes the $s-d$ and $d$ - $d$ exchange interactions in the DMS. Thus the exchange constant in $\mathrm{Cd}_{1-x} \mathrm{Fe}_{x} \mathrm{Te}$, determined from SFRS together with magnetization, is $\alpha N_{0}=244 \pm 10 \mathrm{meV}$, in agreement with the result of excitonic Zeeman experiment. ${ }^{22}$ In addition, the present experiment shows that the $s$ - $d$ exchange interaction is isotropic.

A special feature of SFRS in a Van Vleck DMS is demonstrated in the present paper for $\mathrm{Cd}_{1-x} \mathrm{Fe}_{x} \mathrm{Te}$, similar to that in $\mathrm{Cd}_{1-x} \mathrm{Fe}_{x} \mathrm{Se}^{25}$ viz. a linear relation between the linewidth of SFRS and the $s-d$ exchange strength. Such behavior is explained to be a direct consequence of the nondegenerate nature of the ground state of $\mathrm{Fe}^{2+}$ in II-VI DMSs.

In this work, we also demonstrate, in the context of the induced magnetic moment of $\mathrm{Fe}^{2+}$ in its ground state, the importance of the antiferromagnetic $d$ - $d$ interaction between the nearest neighbor, and, indeed, beyond nearest neighbor, $\mathrm{Fe}^{2+}$ ions for $x \geqslant 0.01$. While the present experiment was limited to the magnetic field of $6 \mathrm{~T}$, SFRS and magnetization

TABLE IV. Probability $P_{2}(x)$ that an $\mathrm{Fe}^{2+}$ ion belongs to a NN pair, an average $g$ factor of $\mathrm{Fe}^{2+}$ at $T=0 \mathrm{~K}$, the molar weight $W(x)$, and the $s$ - $d$ exchange constant $\alpha N_{0}$ for the three $\mathrm{Cd}_{1-x} \mathrm{Fe}_{x} \mathrm{Te}$ samples.

\begin{tabular}{lcccc}
\hline \hline & $P_{2}(x)$ & $\frac{\left\langle\left\langle L_{z}+2 S_{z}\right\rangle\right\rangle}{\left\langle\left\langle S_{z}\right\rangle\right\rangle}$ & $W(x)(g / \mathrm{mol})$ & $\alpha N_{0}(\mathrm{meV})$ \\
\hline $\mathrm{Cd}_{0.991} \mathrm{Fe}_{0.009} \mathrm{Te}$ & 0.09 & 2.32 & 239.5 & $244 \pm 10$ \\
$\mathrm{Cd}_{0.988} \mathrm{Fe}_{0.012} \mathrm{Te}$ & 0.12 & 2.33 & 239.3 & $232 \pm 8$ \\
$\mathrm{Cd}_{0.976} \mathrm{Fe}_{0.024} \mathrm{Te}$ & 0.19 & 2.36 & 238.7 & $207 \pm 10$ \\
\hline \hline
\end{tabular}


measurements at higher fields will certainly uncover the $d$ - $d$ exchange interactions to a greater extent.

\section{ACKNOWLEDGMENTS}

Authors at Purdue thank the National Science Foundation for support through Grant Nos. DMR-0102699 and DMR-
0405082, and Purdue University for support through the Academic Reinvestment Program. The work carried out at the University of North Florida was supported by the Donors of the American Chemical Society Petroleum Research Fund PRF\#40209-B5M, and by National Science Foundation Grant No. DMR-03-05653.
${ }^{1}$ Diluted Magnetic Semiconductors, edited by J. K. Furdyna and J. Kossut, Semiconductors and Semimetals Vol. 25, edited by R. K. Willardson and A. C. Beer (Academic, San Diego, 1988); J. K. Furdyna, J. Appl. Phys. 64, R29 (1988).

${ }^{2}$ H. Munekata, H. Ohno, S. von Molnar, A. Segmüller, L. L. Chang, and L. Esaki, Phys. Rev. Lett. 63, 1849 (1989).

${ }^{3}$ T. Dietl, H. Ohno, F. Matsukura, J. Cibert, and D. Ferrand, Science 287, 1019 (2000).

${ }^{4}$ E. Oh, A. K. Ramdas, and J. K. Furdyna, J. Lumin. 52, 183 (1992)

${ }^{5}$ M. Villeret, S. Rodriguez, and E. Kartheuser, Phys. Rev. B 41, 10028 (1990).

${ }^{6}$ E. Kartheuser, S. Rodriguez, and M. Villeret, Phys. Rev. B 48, 14127 (1993).

${ }^{7}$ S. Tsoi, I. Miotkowski, S. Rodriguez, A. K. Ramdas, H. Alawadhi, and T. M. Pekarek, Phys. Rev. B 69, 035209 (2004).

${ }^{8}$ C. Testelin, C. Rigaux, A. Mauger, A. Mycielski, and C. Julien, Phys. Rev. B 46, 2183 (1992).

${ }^{9}$ D. Colignon, E. Kartheuser, S. Rodriguez, and M. Villeret, Phys. Rev. B 51, 4849 (1995).

${ }^{10}$ G. A. Slack, F. S. Ham, and R. M. Chrenko, Phys. Rev. 152, 376 (1966).

${ }^{11}$ G. A. Slack, S. Roberts, and J. T. Vallin, Phys. Rev. 187, 511 (1969).

${ }^{12}$ J. M. Baranowski and J. M. Langer, Phys. Status Solidi B 48, 863 (1971).

${ }^{13}$ J. P. Mahoney, C. C. Lin, W. H. Brumage, and F. Dorman, J. Chem. Phys. 53, 4286 (1970).

${ }^{14}$ C. Testelin, A. Mauger, C. Rigaux, M. Guillot, and A. Mycielski, Solid State Commun. 71, 923 (1989).

${ }^{15}$ A. Twardowski, D. Heiman, Y. Shapira, T. Q. Vu, and M. Demianiuk, Solid State Commun. 82, 229 (1992).
${ }^{16}$ D. Scalbert, J. A. Gaj, A. Mauger, J. Cernogora, and C. Benoità la Guillaume, Phys. Rev. Lett. 62, 2865 (1989); see also D. Scalbert, A. Mauger, J. A. Gaj, J. Cernogora, M. Nawrocki, and C. Benoità la Guillaume, Phys. Rev. B 43, 7109 (1991).

${ }^{17}$ J. Stühler, R. Meyer, M. Dahl, and G. Schaak, in Proceedings of the 12th International Conference on High Magnetic Fields in the Physics of Semiconductors II, Vol. 2, Würzburg, 1996 (World Scientific, Singapore, 1997), p. 873.

${ }^{18}$ A. Twardowski, P. Glód, W. J. M. de Jonge, and M. Demianiuk, Solid State Commun. 64, 63 (1987).

${ }^{19}$ A. Twardowski, K. Pakula, M. Arciszewska, and A. Mycielski, Solid State Commun. 73, 601 (1990).

${ }^{20}$ C. Testelin, C. Rigaux, A. Mycielski, and M. Menant, Solid State Commun. 78, 659 (1991).

${ }^{21}$ C. Testelin, J. B. Prost, M. Menant, M. Zielinski, and A. Mycielski, Solid State Commun. 113, 695 (2000).

${ }^{22}$ H. Alawadhi, I. Miotkowski, V. Souw, M. McElfresh, A. K. Ramdas, and S. Miotkowska, Phys. Rev. B 63, 155201 (2001).

${ }^{23}$ A. I. Savchuk, B. E. Derkach, O. R. Klichuk, and P. I. Nikitin, IEEE Trans. Magn. 28, 3246 (1992).

${ }^{24}$ X. Wang, C. Chen, A. Li, R. Wang, and K. Ma, J. Appl. Phys. 80, 4421 (1996).

${ }^{25}$ D. Heiman, A. Petrou, S. H. Bloom, Y. Shapira, E. D. Isaacs, and W. Giriat, Phys. Rev. Lett. 60, 1876 (1988).

${ }^{26}$ M. Villeret, S. Rodriguez, and E. Kartheuser, Physica B 162, 89 (1990).

${ }^{27}$ M. K. Udo, M. Villeret, I. Miotkowski, A. J. Mayur, A. K. Ramdas, and S. Rodriguez, Phys. Rev. B 46, 7459 (1992).

${ }^{28}$ D. L. Peterson, D. U. Bartholomew, U. Debska, A. K. Ramdas, and S. Rodriguez, Phys. Rev. B 32, 323 (1985).

${ }^{29}$ C. Testelin, C. Rigaux, A. Mauger, A. Mycielski, and M. Guillot, Phys. Rev. B 46, 2193 (1992). 